\title{
The use of PEEK nanorod arrays for the fabrication of nanoporous surfaces under high temperature: $\mathrm{SiN}_{\mathrm{x}}$ example.
}

\author{
Jaime Martín, Marisol Martín-González* \\ Instituto de Microelectrónica de Madrid (CNM-CSIC), Isaac Newton 8, PTM, E-28760 \\ Tres Cantos, Madrid, Spain \\ marisol@imm.cnm.csic.es
}

\section{Table of contents}

Ordered arrays of poly(ether-ether-ketone) (PEEK) nanopillarshave been fabricated and used as nanomolds for the growth of $\operatorname{SiN}_{\mathrm{x}}$ nanoporous surfaces at $300^{\circ} \mathrm{C}$.

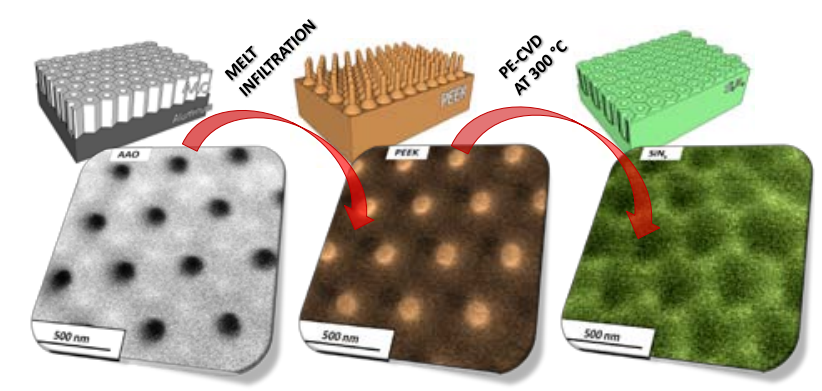

\begin{abstract}
Large area silicon nitride $\left(\mathrm{SiN}_{\mathrm{x}}\right)$ nanoporous surfaces are fabricated using poly(etherether-ketone) (PEEK) nanorod arrays as a templates. The procedure involves manipulation of nanoporous anodic aluminum oxide (AAO) templates in order to form an ordered array of PEEK nanopillars with high temperature resistant characteristics. In this context, self-ordered AAO templates are infiltrated with PEEK melts via the "precursor film” method. Once the melts have been crystallized in the porous structure
\end{abstract}


of AAO, the basis alumina layer is removed, yielding an ordered array of PEEK nanopillars. The resulting structure is a high temperature and chemical resistant polymeric nanomold, which can be utilized in the synthesis of nanoporous materials in aggressive conditions. Such conditions are high temperatures (up to $320^{\circ} \mathrm{C}$ ), vacuum, or extreme $\mathrm{pH}$. For example, $\mathrm{SiN}_{\mathrm{x}}$ nanopore arrays have been grown by plasma enhanced chemical vapor deposition at $300{ }^{\circ} \mathrm{C}$, which can be of interest as mold for nanoimprint lithography, due its hardness and low surface energy. The $\mathrm{SiN}_{\mathrm{x}}$ nanopore array portrays the same characteristics as the original AAO template; $120 \mathrm{~nm}$ diameter pores and an interpore distance of $430 \mathrm{~nm}$. Furthermore, the aspect ratio of the SiNx nanopores can be tuned by selecting an AAO template with appropriate conditions. The use of PEEK as nanotemplate extends the applicability of polymeric nanopatterns into a temperature regime up to now not accessible and opens up the simple fabrication of novel nanoporous inorganic surfaces. 


\section{Introduction}

Ordered structured surfaces with nanoscale lateral dimensions are in high demand for applications in modern technologies ${ }^{1-4}$. Amongst the various means of preparing orderly fashioned surfaces, templates/mold based techniques are becoming the most popular practice due to simplicity, low cost, high process output and high resolution patterning. In this sense, nanoporous Anodic Aluminum Oxide (AAO) has become one of the most important templates due to its characteristic array of uniformly spaced and homogeneous nanopores. The synthetic process of the AAO allows for the control of final length, diameter and latticing parameter of the hexagonal pore arrangement over macroscopic extensions. This entails the tailored preparation of hexagonal arrays of one-dimensional metallic ${ }^{5,6}$, semiconducting ${ }^{7-11}$ or polymeric ${ }^{12,13}$ nanostructures ranging from 20 to $400 \mathrm{~nm}$ in diameter ${ }^{14,15}$ and lengths ranging from hundreds of nanometers to several hundreds of microns. Therefore, it partially solves the limitations of beam lithographies regarding the extension to the nanostructure, and the aspect-ratio (length/diameter) limitations of block-copolymer lithography or nanoimprint lithography. Moreover, ordered arrays of nanopores can be also fabricated through a two-stage replication process from AAO templates ${ }^{16,17}$. For that, the most extended procedure consists of preparing a polymeric negative replica of the AAO template, subsequently using such a negative replica as mold for the fabrication of the nanoporous surface. In fact, when nanostructuring inorganic materials it is often simpler to grow them inside a nanomold, than to directly nanostructure by top-down techniques. However, many of the synthetic procedures of inorganic compounds imply aggressive reaction conditions (high temperature, extreme $\mathrm{pH}$, highly reactive species, etc.), which are hardly supported by most of the commodity polymers the nanomolds are usually

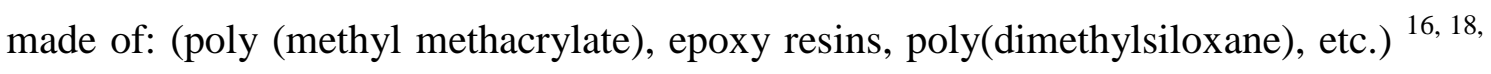
19. Consequently, many inorganic materials cannot be nanostructured by the two-step replication approach.

Thus, the aim of this work is to fabricate ordered arrays of high-resistant polymer nanorods, which can be subsequently used as nanomolds for the growth of inorganic hexagonal nanopore array.

In our approach high performance polymers have been used. High-performancepolymers constitute a special group of polymers that find applications whenever high resistance requirements are needed. Therefore, high-performance-polymers could be 
very attractive for diverse fields of nanotechnology since a nanostructure made of them combines the advantages of commodity polymer nanostructures (the simplicity for being nanostructured, for example) together with the high thermal, mechanical and chemical resistance. We have selected PEEK as our high-performance-polymer model. PEEK is a semi crystalline thermoplastic from the poly(aryl-ether-ketone) family. It presents a continuous serving temperature above $250{ }^{\circ} \mathrm{C}$ and can endure up to $30 \mathrm{~min}$ in an inert atmosphere of up to $400{ }^{\circ} \mathrm{C}^{20}$. Additional properties of PEEK are: high rigidity, low water absorption, high dimensional stability, excellent sliding friction behavior, good electrical characteristics, excellent chemical resistance, low density compared to its metallic and ceramic competitors, and biocompatibility. However, there are very few examples of PEEK nanostructures in scientific literature. Since PEEK is insoluble in all solvents, common polymer nanostructuration techniques such us electrospinning, spin coating, and many forms of nanoprinting techniques are not aplicable methods. Therefore, PEEK must be nanostructured by top-down methodologies or through using melts. Hence, Steinhart and his team successfully created large aspect-ratio PEEK nanotubes by "precursor wetting" of high surface energy hard templates with molten PEEK $^{21,22}$.

In our work, we follow the same strategy for preparing free-standing PEEK nanopillar arrays with a diameter of $120 \mathrm{~nm}$ and aspect ratios up to 3 . In order to show their usability, ordered arrays of silicon nitride $\left(\mathrm{SiN}_{\mathrm{x}}\right)$ nanopores has been prepared by means of Plasma Enhanced Chemical Vapor Deposition (PECVD). In PECVD, PEEK nanopillars are exposed to volatile precursors, which react and/or decompose on the substrate surface to produce the desired deposit at $300{ }^{\circ} \mathrm{C}$. We note that $\operatorname{SiN}_{\mathrm{x}}$ is an interesting material for many applications since it has low density, high temperature strength, high thermal shock resistance, excellent wear resistance, chemical inertness, biocompatibility, and it is a good thermal insulator and dielectric material ${ }^{23}$. And such a $\mathrm{SiN}_{\mathrm{x}}$ nanostructured surface can be of interest for applications in which a high resistant nanostructured surface is required, so other nanoporous materials, such as AAO templates, could not be used (AAO presents poor resistance to acids and alkalis, even diluted). For example, as catalyst support for high temperature or extreme $\mathrm{pH}$ chemical reactions. Furthermore, it could also be suitable as nanomold for nanoimprint lithography thanks to its hardness (increases the life of the nanomold), and thanks to its low surface energy (facilitates the lift off process). It is also widely use in several 
devices, such as bolometric millimeter-wave detectors and lattice microrefrigerators or Bragg-mirrors, since it is a good thermal $\left(k_{\mathrm{SiNx}}=5.5 \mathrm{~W} / \mathrm{m} \cdot \mathrm{K}\right)$ and electrical insulator. When grown by PECVD (as in our case), a non-stechiometric silicon nitride is know to be obtained due to the incorporation of hydrogen atoms into the growing material. Moreover, the amount of hydrogens is inversely proportional to the growth temperature, in such a way that the acceptable compositions are obtained only above $300{ }^{\circ} \mathrm{C}$.

It should be emphasized that this methodology based on PEEK nanostructures can be extrapolated to many materials and techniques that require vacuum, temperatures below $320^{\circ} \mathrm{C}$ or aggressive chemical environments that PEEK is easily able to support.

\section{Experimental part}

\section{Fabrication of AAO templates}

The ordered AAO templates were achieved through the electrochemical anodization of aluminum substrates ${ }^{16,24}$. In this process, 99.999\% pure aluminum foils (Advent Research Materials, England), were cleaned and degreased by sonication with solvents of different polarity (acetone, water, isopropanol, and ethanol). The foils were electropolished in a solution of perchloric acid/ethanol (1/3) under a constant voltage of $20 \mathrm{~V}$, and then, the first anodization was carried out. An aqueous solution of phosphoric acid (1 wt. \%) was used as electrolyte, with a constant acting potential of $205 \mathrm{~V}$. The temperature and length of reaction were $4.5^{\circ} \mathrm{C}$ and $6 \mathrm{~h}$, respectively. Aluminum oxalate was also added to the solution $(0.01 \mathrm{M})$ for stabilizing the reactions as reported

elsewhere 24,25 . The initial anodic layers were then removed through chemical etching in an aqueous solution of phosphoric acid (7 wt. \%) and chromic oxide (1.8 wt. \%). Finally, the second anodization was performed under the same conditions as the first. The lengths of the second anodizations were $90 \mathrm{~s}, 2 \mathrm{~min}$, and $12 \mathrm{~min}$.

\section{Fabrication of PEEK nanopillar arrays}

The used PEEK was purchased from Goodfellow, Ltd. and presented an onset melting temperature of $360{ }^{\circ} \mathrm{C}$ (obtained by differential scanning calorimetry, Fig. 2b). For the PEEK infiltration, the AAO template was cleansed with different polarity 
solvents (water, ethanol, and acetone). Then, the adsorbed organic molecules were removed from the pore walls by heating the AAO template in vacuum. If the organic molecules were not removed, the surface energy of the solid would decrease and the infiltration of the polymer would become difficult. Next, a piece of commercial PEEK was placed onto the surface of the AAO template at a given temperature (well above the melting temperature of the polymer), so the infiltration of the molten PEEK took place by means of the infiltration of precursor films ${ }^{21}$. Experimentally, the infiltration was carried out following these temperature steps (Fig 2a): In the first stage (stage I), the AAO template is heated in a vacuum oven (Brother XD-1200) at the constant heating range of $4{ }^{\circ} \mathrm{C} / \mathrm{min}$ until $390{ }^{\circ} \mathrm{C}$. As mentioned, at high temperature organic molecules are removed from the alumina pore walls. In the second step at $390{ }^{\circ} \mathrm{C}$, a solid piece of PEEK was placed onto the AAO and the system was kept at that temperature for 25 minutes. Finally, the sample was cooled at $3{ }^{\circ} \mathrm{C} / \mathrm{min}$ down to $325{ }^{\circ} \mathrm{C}$ and then at 1 ${ }^{\circ} \mathrm{C} / \mathrm{min}$ down to $250{ }^{\circ} \mathrm{C}$, in order to improve crystallinity. The mechanical, thermal and chemical stability of a polymer material is enhanced in direct correlation with increased crystallinity. All the temperatures were selected according to the melting ( $350^{\circ} \mathrm{C}$ ) and crystallization $\left(\sim 300^{\circ} \mathrm{C}\right)$ temperatures obtained from differential scanning calorimetry (Fig. 2b).

Once the PEEK was crystallized within the nanopores, the polymeric nanopillar array was removed from the AAO template. For that, de aluminum substrate was dissolved in an acidic solution of $\mathrm{CuCl}_{2}$, and the $\mathrm{AAO}$ was dissolved in $\mathrm{NaOH} 10 \mathrm{wt} \%$. Thus, the AAO template is destroyed, which is probably one of the most important drawbacks for a high-throughput fabrication of the process.

\section{Fabrication of $\mathrm{SiN}_{\mathrm{x}}$ nanopore arrays}

The non-stoichiometric $\mathrm{SiN}_{\mathrm{x}}$ film was deposited onto de PEEK nanopillar array by PECVD at $300{ }^{\circ} \mathrm{C}$.

After the deposition of the $\mathrm{SiN}_{\mathrm{x}}$ film on the nanostructured PEEK surface, adhesive cement was placed onto the non-porous surface of the $\operatorname{SiN}_{\mathrm{x}}$. The PEEK was then removed by thermal degradation at $600{ }^{\circ} \mathrm{C}$ in air atmosphere for $4 \mathrm{~h}$, in such a way that the $\mathrm{SiN}_{\mathrm{x}}$ nanoporous film kept supported on the adhesive cement. 


\section{Characterization}

All the prepared samples were morphologically characterized by scanning electron microscopy (SEM) (Hitachi S-800 and Philips XL 30). Before SEM analysis, a gold thin layer was evaporated onto the surface of all samples. During the $\mathrm{Au}$ deposition, the samples (including the PEEK nanopillar arrays) were subjected to high vacuum conditions $\left(10^{-7} \mathrm{mbar}\right)$. The qualitative elemental composition of the $\mathrm{SiN}_{\mathrm{x}}$ nanopore array was evaluated by energy dispersive x-ray spectroscopy (EDX) (Philips XL 30-EDAX.PV 990).

X-ray diffraction (XRD) measurements were carried out using a Philips X'Pert four-circle diffractometer system with $\mathrm{CuK}_{\alpha}$ radiation.

The thermal behavior of bulk PEEK was monitored with a Perkin Elmer DSC-7 differential scanning calorimeter (DSC). Two heating-cooling cycles were carried out at cooling and heating rates of $10 \mathrm{~K} / \mathrm{min}$.

\section{Results}

The $\operatorname{SiN}_{\mathrm{x}}$ nanopore array fabrication method used in this work is based on threesteps (Fig. 1): First, the preparation of a hard template (Fig. 1a), in our case porous alumina AAO with the desired pore diameter. Second, a PEEK melt is infiltrated inside the alumina nanopores (Fig. 1b) in order to obtain a PEEK nanopillar array after the removal of the AAO (Fig. 1c). Third, the PEEK nanorod array is used as a second mold for the nanostructured growth of the $\operatorname{SiN}_{x}$ (Fig. 1d). Finally, the PEEK is removed and a $\mathrm{SiN}_{\mathrm{x}}$ film with the same morphology as that of the original AAO is obtained (Fig 1e).

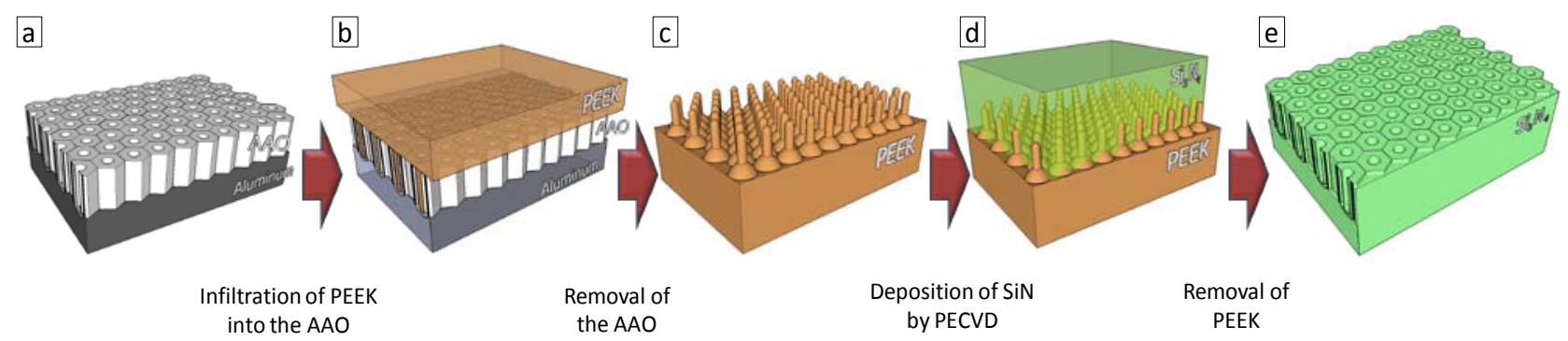

Fig. 1. Schematic diagram of the two-step replication process of nanoporous alumina surface to obtain a nanopore array made of the silicon nitride $\left(\mathrm{SiN}_{\mathrm{x}}\right)$. First nanoporous hard template of 
Anodic Aluminum Oxide (AAO) is synthesized (a); then the molten poly(ether-ether-ketone) (PEEK) is infiltrated into the nanopores by means of the "precursor film" infiltration method (b) and after removal of the template, a PEEK nanopillar array is obtained (c). The nanopillar array is used as a negative mold for the preparation of a replica of the original AAO template. In this

step, the $\mathrm{SiN}_{\mathrm{x}}$ is grown by plasma enhanced chemical vapor deposition (PECVD) on the nanopillar array (d). Finally, the PEEK is thermally removed giving rise to an ordered array of $\mathrm{SiN}_{\mathrm{x}}$ nanopores (e).

The AAO templates obtained exhibited a polydomain hexagonal array of cylindrical nanopores, in which the pores were $120 \mathrm{~nm}$ in diameter and the interpore distance was $430 \mathrm{~nm}$ (as observed in Fig.3). Pores with lengths of around $100 \mathrm{~nm}$ (aspect-ratio of 1), $350 \mathrm{~nm}$ (aspect-ratio of 3) and $1000 \mathrm{~nm}$ (aspect-ratio of 8) were obtained in the AAOs anodized for 90 s, 2 min and 12 min, respectively (Fig. 2).

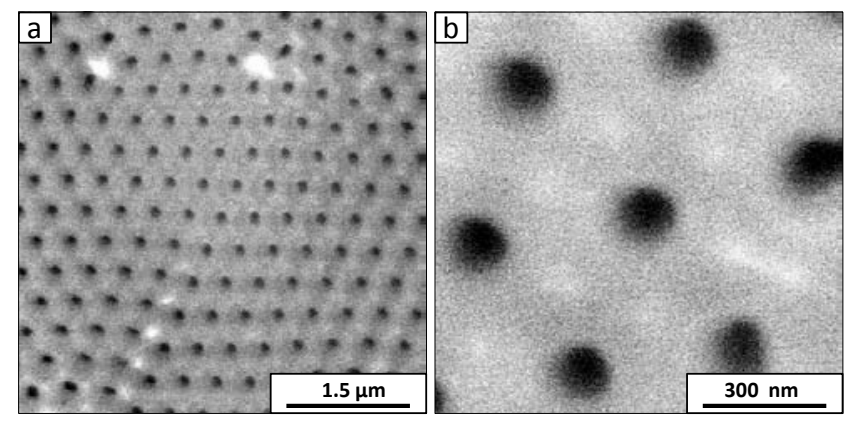

Fig 2. (a) Low magnification and (b) high magnification SEM micrographs of the surface of the AAO template with $120 \mathrm{~nm}$ diameter pores.

The subsequent infiltration of the PEEK into the AAO nanopores was achieved by the "precursor film infiltration" $\operatorname{method}^{12}$, based on the wetting behavior of low surface energy liquids (molten PEEK) onto high surface energy solids (hydroxilated AAO pore walls). When both components are placed in contact under certain temperature conditions, the liquid PEEK precursor films spontaneously spread to wet the AAO pore walls, hence the infiltration process ${ }^{26}$. Experimentally, infiltration was carried at $390^{\circ} \mathrm{C}$ (Fig. 3a, Step II). All the temperatures were selected according to the 
melting peak temperature $\left(\sim 360{ }^{\circ} \mathrm{C}\right)$ and onset crystallization temperature $\left(\sim 310{ }^{\circ} \mathrm{C}\right)$ obtained from differential scanning calorimetry (Fig. 3b).
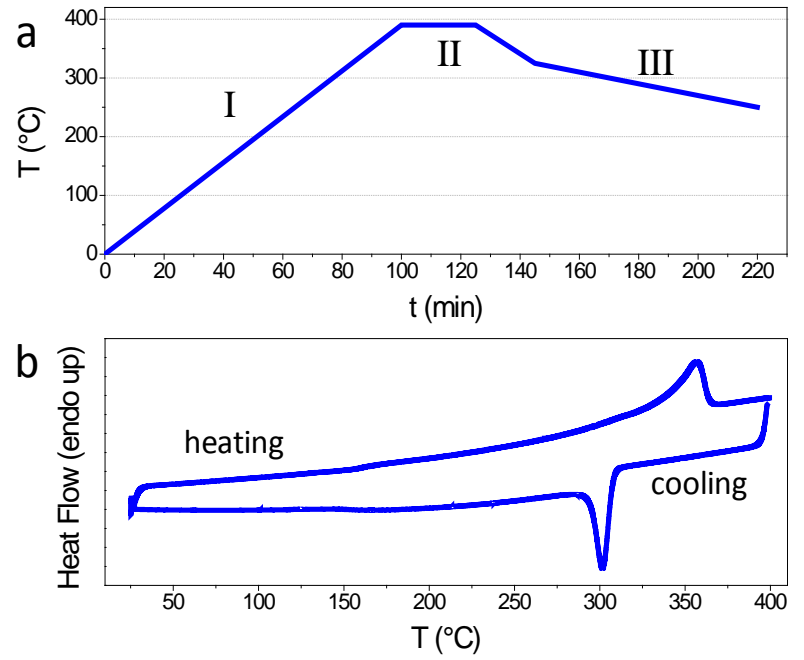

Fig 3. (a) Applied temperature program for the infiltration of molten PEEK into the nanopores. (I) The AAO is heated up to the infiltration temperature. (II) The infiltration takes place at a constant temperature. (III) Crystallization of the PEEK nanostructure. (b) Differential scanning calorimetry runs for PEEK.
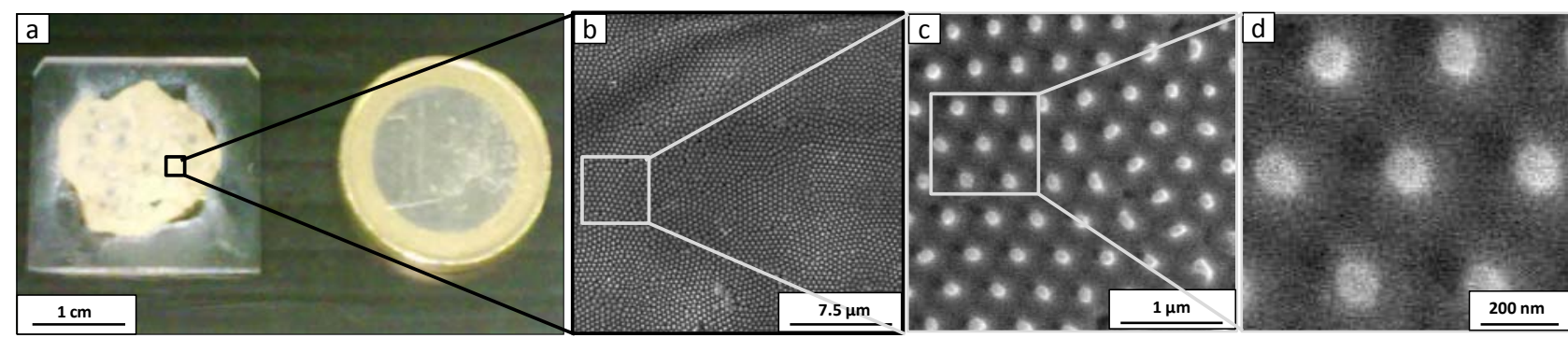

Fig. 4. (a) Photograph of the PEEK nanopillar array (right) together with a $1 €$ coin (left) for comparison. (b, c, and d) SEM micrographs of the surface of the PEEK nanopillar array at different magnifications.

Fig. 4 shows the obtained PEEK nanopillar array after the removal of the AAO template. The SEM micrographs of the nanostructure (Fig. 4b-d) demonstrate that the AAO pattern has been perfectly transferred to the polymeric material in such a way that 
structural defects are absent in the new pattern. Thus, the PEEK pillar array displays a hexagonal closed-packed symmetry over domains exceeding 10 inter-pillar distances. On a larger scale, the typical polydomain structure of the AAO can be observed in Fig. $4 \mathrm{~b}$, in which the nanopillars are120 nm in diameter. Finally, the inter-pillar distance is $430 \mathrm{~nm}$, therefore the density of the nanopillars is of the order $10^{9}$ pillar $/ \mathrm{cm}^{2}$. Moreover, the nanostructured area extends over $\mathrm{cm}^{2}$, as can be observed in the photograph shown in Fig. 4a.

The semi crystalline nature of the PEEK after the nanostructuration process was confirmed by wide angle X-Ray diffraction (XRD) (Fig. 5). The nanostructured PEEK sample shows the characteristic diffraction maxima centered at $2 \theta=18.8^{\circ}, 20.7^{\circ} 22.8^{\circ}$, and $28.8^{\circ}$, corresponding to $d$ spacings of 4.72, 4.28, 3.89, and $3.09 \AA$. Those spacings can be associated with the (110), (111), (200), and (211) planes ${ }^{27}$, respectively.

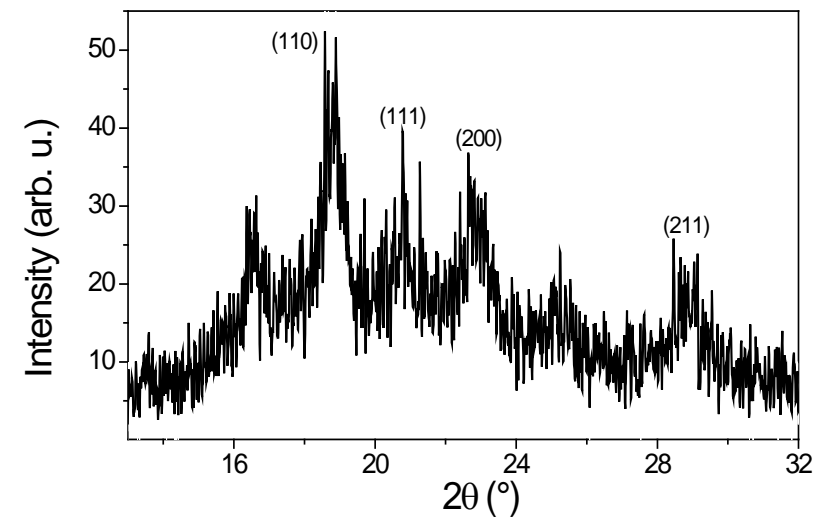

Fig. 5. XRD pattern of the nanostructured PEEK sample after infiltration. 


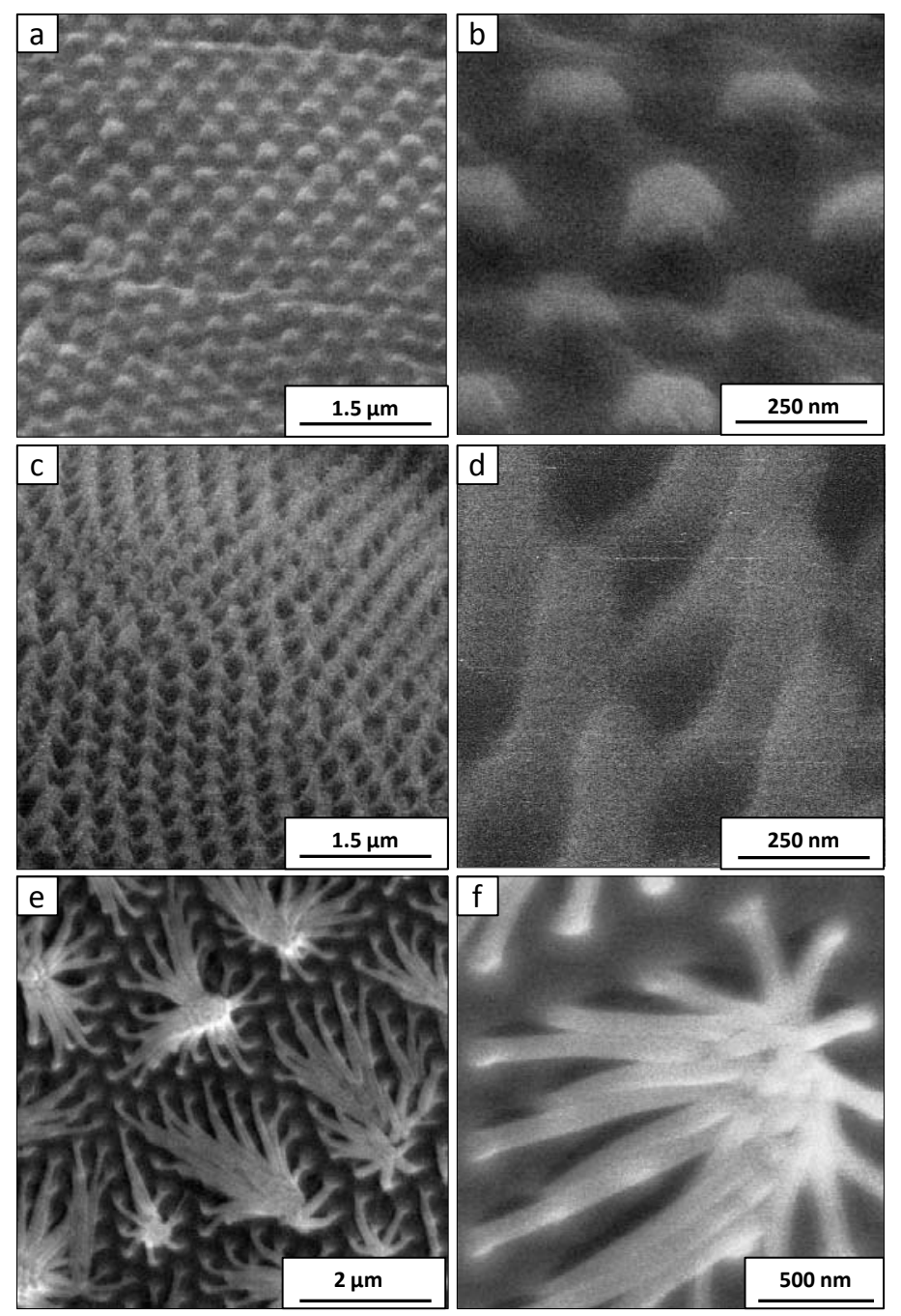

Fig. 6. Ordered arrays of PEEK nanopillars with different aspect ratios: (a) and (b) aspect-ratio closed to 1; (c) and (d) aspect-ratio around 3; and (e) and (f) aspect-ratio around 8. The highest aspect ratio before the PEEK nanorods fold down is below 8 for PEEK nanopillars.

Moreover, the characteristic dimensions of the nanostructure can be tailored simply by selecting the appropriate AAO template. In Fig. 6, different aspect-ratio nanopillars are displayed. Fig. 6a and 6b correspond to PEEK nanopillars with an aspect ratio close to 1 , which have been prepared from an AAO having pores of the same aspect-ratio. Fig. 6c and 6d show nanopillars with aspect-ratio around 3, while the nanopillars in Fig. 6e and $6 \mathrm{f}$ have an aspect-ratio of 8 . As can be observed, nanopillars having such a high aspect-ratio are aggregated in bundles so the ordered nanostructure is lost. Therefore, pillars having this aspect-ratio are not valid for obtaining ordered porous surfaces. The highest aspect ratio before folding down is frequently below 5 for 
polymer nanopillars unless they have been mechanically extracted from the porous AAO template ${ }^{17}$, or dried under special conditions ${ }^{28}$.

Apart from the simplicity of its nanostructuration process, the most attractive quality of a high-performance-polymeric nanostructure is its high resistance to extreme conditions. Our fabricated PEEK nanopillar arrays maintain their morphological integrity without apparent damage in the nanostructure, when exposed to a temperature of $300{ }^{\circ} \mathrm{C}$ for $60 \mathrm{~min}$ (Fig. 7). Moreover, they are also stable under high vacuum as evident in Fig. 4 and Fig. 5, where the PEEK nanopillar arrays had been subjected to $10^{-}$ ${ }^{7}$ mbar during the Au evaporation performed for the SEM analysis. Overall, the PEEK nanopillar arrays have been shown to be compatible with high temperature and high vacuum processes, and can be used as a template for the growth of a secondary nanostructure by means of high temperature or high vacuum synthetic procedures.
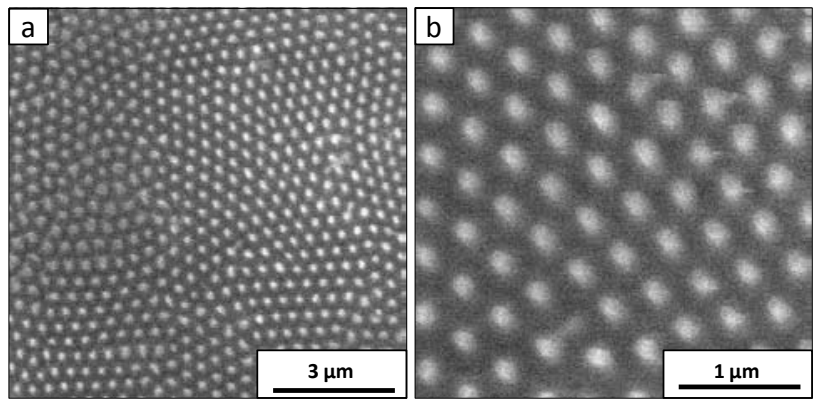

Fig. 7. Surface view of the PEEK nanostructure after being thermally annealed at $300{ }^{\circ} \mathrm{C}$ for 60 min.

In order to demonstrate the usability of the PEEK nanopillar array, we fabricated an ordered array of $\mathrm{SiN}_{\mathrm{x}}$ nanopores mimicking the nanostructure of the AAO templates. It is worth emphasizing that the synthesis of the $\mathrm{SiN}_{\mathrm{x}}$ by means of PECVD was carried out onto the PEEK nanostructure at $300{ }^{\circ} \mathrm{C}$. 

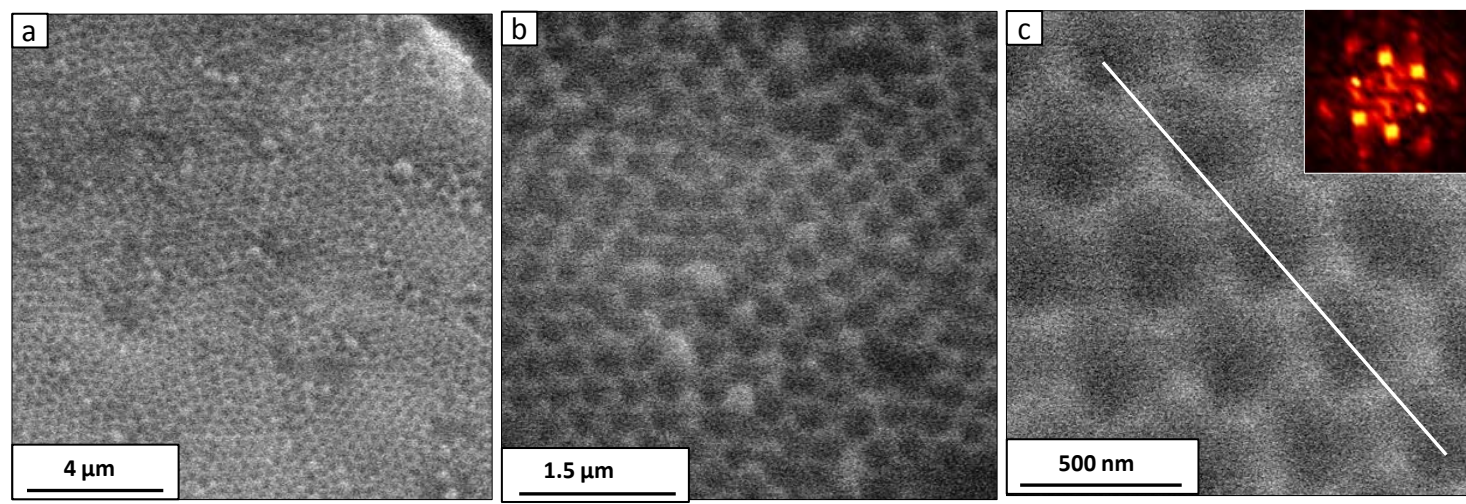

d

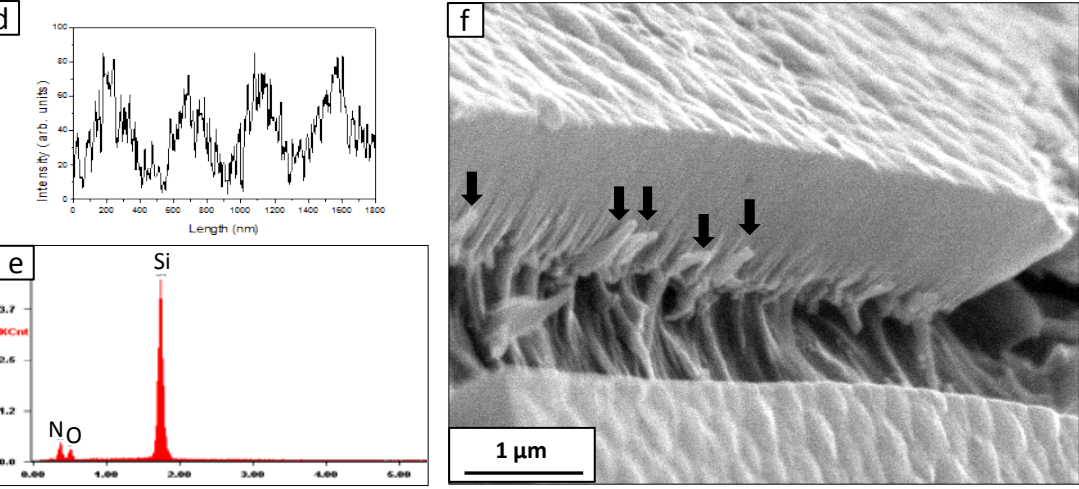

Fig. 8. Ordered arrays of $\operatorname{SiN}_{\mathrm{x}}$ nanopores. (a, b, and c) SEM micrographs of the surface at different magnifications. The inset in (c) corresponds to the Fourier transform of the image, where the hexagonal symmetry of the pore arrangement is evidenced. (d) Surface intensity profile measured along the straight line drawn in (c). (e) Representative EDX spectrum of the

$\mathrm{SiN}_{\mathrm{x}}$ pore array. (f) Cross section of $\mathrm{SiN}_{\mathrm{x}}$ film with pores of aspect ratio equal to 3 still deposited on the PEEK nanopillars. PEEK nanopillars (marked with arrows) can be distinguished protruding from the broken $\mathrm{SiN}_{\mathrm{x}}$ pores.

Fig 8a, 8b, and 8c show SEM micrographs of the obtained $\mathrm{SiN}_{\mathrm{x}}$ nanopore array. The PEEK pattern has been completely transferred to the $\mathrm{SiN}_{\mathrm{x}}$, in such a way that the pores are distributed according to a honeycomb nanostructure, as evidenced in the Fourier transform of the image (c) (Fig. 8c inset). During the deposition, the $\mathrm{SiN}_{\mathrm{x}}$ not only covers the surface of the nanopillars but also fills the hollow space between them, giving rise to a continuous $\mathrm{SiN}_{\mathrm{x}}$ film of $1 \mu \mathrm{m}$ thickness (Fig 8f). The dimensions of the new nanostructure are identical to that of the used AAO template: the pores are around $120 \mathrm{~nm}$ in diameter and the interpore distance is $430 \mathrm{~nm}$, as shown in the surface profile in Fig 8d. The profile corresponds to the color intensity profile along the straight line drawn in Fig. 8c. The qualitative elemental composition of the $\operatorname{SiN}_{\mathrm{x}}$ nanopore array is shown in the energy dispersive X-ray (EDX) analysis (Fig. 8e). The EDX spectrum evidences that the obtained $\operatorname{SiN}_{\mathrm{x}}$ nanopore array is Si-rich. The spectrum shown is representative of the whole film. The $\mathrm{SiN}_{\mathrm{x}}$ nanopores shown in Fig. 8a, 8b, and $8 \mathrm{c}$ have 
an aspect ratio of around 1, while the cross section view in Fig. 8f corresponds to a $\operatorname{SiN}_{\mathrm{x}}$ film with a pore aspect ratio of 3, still deposited on the PEEK nanopillars. Such PEEK nanopillars can be identified protruding from the broken $\mathrm{SiN}_{\mathrm{x}}$ nanopores (marked with an arrow).

It is important to highlight that the $\operatorname{SiN}_{\mathrm{x}}$ nanopore array fabrication process presented in this work is notably simple and economic, because it does not involve a costly lithographic processes. Moreover, both the patternable area and the structural dimensions of the nanopore array are directly given by those of the original hard template, so, areas of $\mathrm{cm}^{2}$ and nanopores ranging from few tens of $\mathrm{nm}$ up to several hundreds of $\mathrm{nm}$ can be obtained. Thus, our $\mathrm{SiN}_{\mathrm{x}}$ nanopore array could be interesting for applications where a high-performance nanoporous surface is required: The high temperature strength, high thermal shock resistance, and chemical inertness of the $\mathrm{SiN}_{\mathrm{x}}$, together with the high specific surface of the nanopore array, make this material attractive for high temperature catalist support, for example. Furthermore, it could be also adequate as nanomold for nanoimprint lithography due to its hardness, which results in a longer useful life of the nanomold; and due to its low surface energy, which facilitates the lift off process.

\section{Conclusions}

An efficient and simple methodology for the fabrication of ordered nanopore arrays has been developed to be used in extreme conditions. The procedure is based on a twostage replication of alumina templates. First, we prepared a free-standing hexagonal array of semicrystalline PEEK nanopillars by wetting AAO templates with PEEK melts. Afterwards, the obtained PEEK nanopillar array is used as a nanomold for the growth of the $\mathrm{SiN}_{\mathrm{x}}$ nanopore array by means of PECVD at $300{ }^{\circ} \mathrm{C}$. Hence, the polymeric nanostructure becomes stabilized at such a high temperature. The morphology of the PEEK is completely transferred to the $\mathrm{SiN}_{\mathrm{x}}$, in such a way that the $\mathrm{SiN}_{\mathrm{x}}$ surface is almost a replica of that of the original AAO template. Thus, its nanopores are $120 \mathrm{~nm}$ in diameter and the interpore distance is $430 \mathrm{~nm}$. The aspect ratios of the obtained nanopores were 1 and 3 , and can be adjusted by selecting the appropriate AAO 
template. The fabrication process of the $\mathrm{SiN}_{\mathrm{x}}$ nanopore array is notably economic and allows obtaining areas of $\mathrm{cm}^{2}$. However, the AAO is destroyed during the process, which could hinder the high-throughput production of nanoporous surfaces via this method. One possible solution could be to reduce the surface energy of the original AAO pore wall with some kind of material or compound stable at the PEEK infiltration temperature, so that afterwards, the PEEK nanopillars can be mechanically extracted from the nanopores without disolving the AAO template.The used methodology can be extrapolated to other many materials and techniques that require vacuum, temperatures $<320^{\circ} \mathrm{C}$ or aggressive chemical environments.

\section{Acknowledgments}

Authors want to thank the ERC 2008 Starting Grant "Nano-TEC” number 240497 for financial support. We acknowledge R. Alvaro for the $\mathrm{SiN}_{\mathrm{x}}$ deposition and Yazmin Feliz for the idiomatic corrections of the manuscript. 


\section{References}

1. E. Chow, S. Y. Lin, S. G. Johnson, P. R. Villeneuve, J. D. Joannopoulos, J. R. Wendt, G. A. Vawter, W. Zubrzycki, H. Hou and A. Alleman, Nature, 2000, 407, 983-986.

2. C. Ross, Annual Review of Materials Research, 2001, 31, 203-235.

3. S. Grimm, J. Martin, G. Rodriguez, M. Fernandez-Gutierrez, K. Mathwig, R. B. Wehrspohn, U. Gösele, J. San Román, C. Mijangos and M. Steinhart, Journal of Materials Chemistry, 2010, 20, 3171-3177.

4. Z. Hu, M. Tian, B. Nysten and A. M. Jonas, Nat Mater, 2009, 8, 62-67.

5. B. Luo, D. Yang, M. Liang and L. Zhi, Nanoscale, 2010, 2, 1661-1664.

6. C. R. Martin, Science, 1994, 266, 1961-1966.

7. M. Martín-González, A. L. Prieto, R. Gronsky, T. Sands and A. M. Stacy, Advanced Materials, 2003, 15, 1003-1006.

8. M. Martín-González, G. J. Snyder, A. L. Prieto, R. Gronsky, T. Sands and A. M. Stacy, Nano Letters, 2003, 3, 973-977.

9. M. Martín-González, A. L. Prieto, M. S. Knox, R. Gronsky, T. Sands and A. M. Stacy, Chemistry of Materials, 2003, 15, 1676-1681.

10. A. L. Prieto, M. S. Sander, M. S. Martín-González, R. Gronsky, T. Sands and A. M. Stacy, Journal of the American Chemical Society, 2001, 123, 7160-7161.

11. L. Shi, Y. Xu and Q. Li, Nanoscale, 2010, 2, 2104-2108.

12. M. Steinhart, J. H. Wendorff, A. Greiner, R. B. Wehrspohn, K. Nielsch, J. Schilling, J. Choi and U. Gösele, Science, 2002, 296, 1997.

13. J. Martín and C. Mijangos, Langmuir, 2008, 25, 1181-1187.

14. J. Martín, C. V. Manzano and M. Martín-González, Microporous and Mesoporous Materials, 2012, 151, 311-316.

15. O. Jessensky, F. Muller and U. Gosele, Appl. Phys. Lett., 1998, 72, 1173-1175.

16. H. Masuda and K. Fukuda, Science, 1995, 268, 1466-1468.

17. S. Grimm, R. Giesa, K. Sklarek, A. Langner, U. Gösele, H.-W. Schmidt and M. Steinhart, Nano Letters, 2008, 8, 1954-1959.

18. B. D. Gates, Q. Xu, M. Stewart, D. Ryan, C. G. Willson and G. M. Whitesides, Chemical Reviews, 2005, 105, 1171-1196.

19. D. Navas, M. Hernandez-Velez, M. Vazquez, W. Lee and K. Nielsch, Appl. Phys. Lett., 2007, 90, 192501.

20. A. Jonas and R. Legras, Polymer, 1991, 32, 2691-2706.

21. M. Steinhart, Advances in Polymer Science, 2008, 123-187.

22. M. Steinhart, R. B. Wehrspohn, U. Gösele and J. H. Wendorff, Angewandte Chemie International Edition, 2004, 43, 1334-1344.

23. Y. Shi, Y. Wan and D. Zhao, Chemical Society Reviews, 2011, 40, 3854-3878.

24. C. Sun, J. Luo, L. Wu and J. Zhang, ACS Applied Materials \& Interfaces, 2010, 2, 12991302.

25. J. Martín, M. Martín-González, A. d. Campo, J. J. Reinosa and J. F. Fernández, in preparation, 2012.

26. J. Martín, J. Maiz, J. Sacristan and C. Mijangos, Polymer, 2012, 53, 1149-1166.

27. P. C. Dawson and D. J. Blundell, Polymer, 1980, 21, 577-578.

28. N. Haberkorn, J. S. Gutmann and P. Theato, ACS Nano, 2009, 3, 1415-1422. 